Constructive existence analysis of solutions of non-linear integral boundaryvalue problems

Miklós Rontó and Yana Varha 


\title{
CONSTRUCTIVE EXISTENCE ANALYSIS OF SOLUTIONS OF NON-LINEAR INTEGRAL BOUNDARY VALUE PROBLEMS
}

\author{
MIKLÓS RONTÓ AND YANA VARHA
}

Received 08 September, 2014

\begin{abstract}
Sufficient conditions guaranteeing the solvability of non-linear integral boundary value problems for a system of non-linear ordinary differential equations are obtained using a special successive approximation technique. The efficiency of the suggested approach is shown on an example of a non-linear integral boundary value problem possessing at least two solutions.
\end{abstract}

2010 Mathematics Subject Classification: 34B15

Keywords: Non-linear system of differential equations, integral boundary conditions, parametrization technique, successive approximations, existence

\section{INTRODUCTION}

In [9], a new approach has been suggested for the investigation of existence and approximate construction of solutions of non-local boundary value problems for ordinary differential equations. The purpose of the present paper is to apply this technique to obtain a scheme for the constructive solvability analysis of integral boundary value problems in the case where the non-local boundary conditions depend involve both the space variable and its derivative. Note that our approach is easier to apply compared with those used earlier [3, 4, 6, 12,14,15] for more special cases. At first, the given problem is reduced to a certain "model-type" one with a very simple twopoint separated linear boundary condition depending on parameters. The transformed problem is then replaced by the Cauchy problem for suitably perturbed system containing some artificially introduced vector parameters the numerical values of which are to be determined later. The functional perturbation term, together with the given integral boundary conditions, generates a system of finite-dimensional system of algebraic or transcendental "determining" equations from which the numerical values of introduced parameters should be found. The solvability of the determining system, in turn, may be checked by studying some approximations constructed explicitly. We use for that purpose topological degree techniques similarly to $[6,7,15]$. 


\section{NOTATION AND SYMBOLS}

We will use mainly the notation from [9]. For any vector $x=\operatorname{col}\left(x_{1}, \ldots, x_{n}\right) \in \mathbb{R}^{n}$, the obvious notation $|x|=\operatorname{col}\left(\left|x_{1}\right|, \ldots,\left|x_{n}\right|\right)$ is used and the inequalities between vectors are understood componentwise. The same convention is adopted implicitly for operations "max", "min", etc. applied to vector-valued functions. The symbol $\mathbb{1}_{n}$ stands for the unit matrix of dimension $n$ and $r(K)$ denotes the maximal, in modulus, eigenvalue of a square matrix $K$.

Definition 1. For any non-negative vector $\rho \in \mathbb{R}^{n}$ under the componentwise $\rho$ neighbourhood of a point $z \in \mathbb{R}^{n}$ we understand the set

$$
B(z, \rho):=\left\{\xi \in \mathbb{R}^{n}:|\xi-z| \leq \rho\right\} .
$$

Similarly, for the given bounded connected set $\Omega \subset \mathbb{R}^{n}$, we define its componentwise $\rho$-neighbourhood by putting

$$
B(\Omega, \rho):=\bigcup_{\xi \in \Omega} B(\xi, \rho) .
$$

Definition 2. For given two bounded connected sets $D_{a} \subset \mathbb{R}^{n}$ and $D_{b} \subset \mathbb{R}^{n}$, introduce the set

$$
D_{a, b}:=\left\{(1-\theta) z+\theta \eta: z \in D_{a}, \eta \in D_{b}, \theta \in[0,1]\right\}
$$

and its componentwise $\rho$-neighbourhood

$$
D:=B\left(D_{a, b}, \rho\right) .
$$

For a set $D \subset \mathbb{R}^{n}$, closed interval $[a, b] \subset \mathbb{R}$, Carathéodory function $f:[a, b] \times$ $D \rightarrow \mathbb{R}^{n}, n \times n$ matrix $K$ with non-negative entries, we write

$$
f \in \operatorname{Lip}_{K}(D)
$$

if the inequality

$$
|f(t, u)-f(t, v)| \leq K|u-v|
$$

holds for all $\{u, v\} \subset D$ and a. e. $t \in[a, b]$.

Finally, with a function $f:[a, b] \times D \rightarrow \mathbb{R}^{n}$, we associate the vector

$$
\delta_{[a, b], D}(f):=\frac{1}{2}\left(\operatorname{esssup}_{(t, x) \in[a, b] \times D} f(t, x)-\operatorname{essinf}_{(t, x) \in[a, b] \times D} f(t, x)\right) .
$$

\section{PRoblem SETting AND REDUCTION TO A MODEL BOUNDARY CONDITION}

We consider the following non-linear integral boundary value problem which was studied in [13]

$$
\begin{gathered}
\frac{d x}{d t}=f(t, x), \quad t \in[a, b] \\
\int_{a}^{b}[g(s, x(s))+h(s, f(s, x(s)))] d s=d .
\end{gathered}
$$


Let $D_{a}$ and $D_{b}$ be a convex subsets of $\mathbb{R}^{n}$ where one looks for respectively the initial value $x(a)$ and the value $x(b)$ of the solution of the boundary value problem (3.1), (3.2).

Based on the sets $D_{a}$ and $D_{b}$ according to (2.1) we introduce the convex set $D_{a, b}$ and its componentwise $\rho$-neighbourhood $D$ as in (2.2). Thus, the domain of the space variables in the given problem (3.1), (3.2) is the set $D$ defined according to (2.2).

Here we suppose that the functions $f:[a, b] \times D \rightarrow \mathbb{R}^{n}, g:[a, b] \times D \rightarrow \mathbb{R}^{n}$, and $h:[a, b] \times D \rightarrow \mathbb{R}^{n}$ satisfy the Carathéodory and the Lipschitz condition in the domain $D$ and $d$ is a given vector.

Finally we suppose that the vector $\rho$ in (2.2) is chosen so that

$$
\rho \geq \frac{b-a}{2} \delta_{[a, b], D}(f),
$$

where $\delta_{[a, b], D}(f)$ is given in (2.4). We also assume that the maximal in modulus eigenvalue of the matrix

$$
Q:=\frac{3(b-a)}{10} K
$$

satisfies the inequality

$$
r(Q)<1 .
$$

It is important to emphasize that $D \subset \mathbb{R}^{n}$ is supposed to be bounded and, thus, the Lipschitz condition is not assumed globally.

The problem is to find and establish the existence of an absolutely continuous solution $x:[a, b] \rightarrow D$ of the problem (3.1), (3.2) with initial value $x(a) \in D_{a}$.

At first we simplify the boundary condition (3.2) and reduce it to a suitable twopoint separated linear one. To do so, similarly to $[5,10,11,14]$ we apply an appropriate "freezing" technique. Namely, we introduce the vectors of parameters

$$
z=\operatorname{col}\left(z_{1}, z_{2}, \ldots, z_{n}\right), \quad \eta=\operatorname{col}\left(\eta_{1}, \eta_{2}, \ldots, \eta_{n}\right)
$$

by formally putting

$$
z=x(a), \quad \eta=x(b) .
$$

Now, instead of integral problem (3.1), (3.2) we will consider the following "modeltype" two-point boundary value problem with separated parameterized conditions:

$$
\begin{array}{cc}
\frac{d x}{d t}=f(t, x), & t \in[a, b], \\
x(a)=z, & x(b)=\eta .
\end{array}
$$

The parameterization technique that we are going to use suggest that, instead of the original boundary value problem with nonlinear integral boundary conditions (3.2), we study the family of parametrized boundary value problems (3.8), (3.9), where the boundary restrictions are linear and separated. Later, we return to the original problem by choosing the values of the introduced parameters appropriately. 
Remark 1. The set of solutions of the non-linear integral boundary value problem (3.1), (3.2) coincides with the set of the solutions of the parametrized problem (3.8), (3.9) with separated restrictions, satisfying additional conditions (3.9).

\section{SOME RESUlTS FROM [13]}

Similarly to $[9,13]$ let us associate with the two-point parametrized boundary value problem (3.8), (3.9) with separated boundary conditions the sequence of functions

$$
\begin{aligned}
x_{m+1}(t, z, \eta)=z & +\int_{a}^{t} f\left(s, x_{m}(s, z, \eta)\right) d s-\frac{t-a}{b-a} \int_{a}^{b} f\left(\tau, x_{m}(\tau, z, \eta)\right) d \tau \\
& +\frac{t-a}{b-a}[\eta-z], \quad t \in[a, b], \quad m=1,2, \ldots,
\end{aligned}
$$

satisfying (3.9) for arbitrary $z, \eta \in \mathbb{R}^{n}$, where

$$
x_{0}(t, z, \eta)=z+\frac{t-a}{b-a}[\eta-z]=\left(1-\frac{t-a}{b-a}\right) z+\frac{t-a}{b-a} \eta, \quad t \in[a, b],
$$

and $z \in D_{a}, \eta \in D_{b}$ are considered as parameters.

It is obvious from (4.2) that $x_{0}(t, z, \eta)$ is a convex combination of vectors $z$ and $\eta$ for any $t \in[a, b]$.

The following Theorems 1, 2, and 3 were proved in [13].

Theorem 1 ([13, Theorem 1]). Let there exists a non-negative vector $\rho$ such that inequality (3.3) holds and $f \in \operatorname{Lip}_{K}(D)$ for $D$ given by (2.2) with the given value of $\rho$. Let, moreover, the matrix $K$ be such that (3.5) holds with $Q$ given by (3.4). Then, for all fixed $(z, \eta) \in D_{a} \times D_{b}$ :

1. The functions of sequence (4.1) are absolutely continuous on $[a, b]$, satisfy the two-point separated boundary conditions (3.9), and have values in $D$.

2. The sequence of functions (4.1) converges as $m \rightarrow \infty$ to the limit function

$$
x_{\infty}(t, z, \eta):=\lim _{m \rightarrow \infty} x_{m}(t, z, \eta)
$$

uniformly in $t \in[a, b]$.

3. The limit function satisfies the initial condition

$$
x_{\infty}(a, z, \eta)=z
$$

and the two-point separated boundary conditions (3.9).

4. The function $x_{\infty}(\cdot, z, \eta)$ is a unique absolutely continuous solution of the integral equation

$$
x(t)=z+\int_{a}^{t} f(s, x(s)) d s-\frac{t-a}{b-a} \int_{a}^{b} f(s, x(s)) d s+\frac{t-a}{b-a}[\eta-z] .
$$


In other words, $x_{\infty}(\cdot, z, \eta)$ satisfies the Cauchy problem for the modified system of integro-differential equations:

$$
\begin{gathered}
\frac{d x}{d t}=f(t, x)+\frac{1}{b-a} \Delta(z, \eta), \quad t \in[a, b], \\
x(a)=z,
\end{gathered}
$$

where $\Delta: D_{a} \times D_{b} \rightarrow \mathbb{R}^{n}$ is the mapping given by formula

$$
\Delta(z, \eta):=\eta-z-\int_{a}^{b} f\left(s, x_{\infty}(s, z, \eta)\right) d s .
$$

5. The following error estimate holds:

$$
\left|x_{\infty}(t, z, \eta)-x_{m}(t, z, \eta)\right| \leq \frac{10}{9} \alpha_{1}(t, a, b) Q^{m}\left(\mathbb{1}_{n}-Q\right)^{-1} \delta_{[a, b], D}(f),
$$

for any $t \in[a, b]$ and $m \geq 0$, where $\delta_{[a, b], D}(f)$ is given in (2.4) and

$$
\alpha_{1}(t, a, b):=2(t-a)\left(1-\frac{t-a}{b-a}\right) .
$$

Remark 2. It is easy to verify that $\max _{t \in[a, b]} \alpha_{1}(t, a, b)=(b-a) / 2$.

Theorem 2 ([13, Theorem 3]). Let $(z, \eta) \in D_{a} \times D_{b}$. Under the assumptions of Theorem 1, the limit function $x_{\infty}(\cdot, z, \eta)$ of sequence (4.1) is an absolutely continuous solution of the integral boundary value problem (3.1), (3.2) if and only if the pair of parameters $(z, \eta)$ satisfies the system of $2 n$ algebraic determining equations

$$
\begin{aligned}
& \Delta(z, \eta)=0, \\
& \Lambda(z, \eta)=0,
\end{aligned}
$$

with $\Delta: D_{a} \times D_{b} \rightarrow \mathbb{R}^{n}$ given by formula (4.4) and

$$
\Lambda(z, \eta):=\int_{a}^{b}\left[g\left(s, x_{\infty}(s, z, \eta)\right)+h\left(s, f\left(s, x_{\infty}(s, z, \eta)\right)\right)\right] d s-d .
$$

The next statement proves that the system of determining equations (4.7), (4.8) defines all possible solutions of the original non-linear integral boundary value problem (3.1), (3.2).

Theorem 3 ([13, Theorem 4]). Assume that conditions of Theorem 1 is satisfied. If there exists a pair of vectors $\left(z^{0}, \eta^{0}\right) \in D_{a} \times D_{b}$ satisfying the system of determining equations (4.7), (4.8), then the integral boundary value problem (3.1), (3.2) has a solution $x^{0}(\cdot)$ such that

$$
x^{0}(a)=z^{0}, \quad x^{0}(b)=\eta^{0}
$$

and, moreover, $x^{0}(\cdot)=x_{\infty}\left(\cdot, z^{0}, \eta^{0}\right)$.

Conversely, if the integral boundary value problem (3.1), (3.2) has a solution $x^{0}(\cdot)$ with $\left(x^{0}(a), x^{0}(b)\right) \in D_{a} \times D_{b}$ and $\left\{x^{0}(t): t \in[a, b]\right\} \subset D$, then $x^{0}(\cdot)$ necessarily 
has the form $x^{0}(\cdot)=x_{\infty}\left(\cdot, x^{0}(a), x^{0}(b)\right)$, and the system of determining equations (4.7), (4.8) is satisfied with $z=x^{0}(a), \eta=x^{0}(b)$.

\section{SOLVABILITY ANALYSIS BASED ON THE APPROXIMATE DETERMINING SYSTEM}

The solvability of the determining system (4.7), (4.8), in turn, may be checked by using the so-called approximate determining equations

$$
\begin{aligned}
& \Delta_{m}(z, \eta)=0, \\
& \Lambda_{m}(z, \eta)=0,
\end{aligned}
$$

where $m$ is fixed and

$$
\begin{aligned}
& \Delta_{m}(z, \eta):=\eta-z-\int_{a}^{b} f\left(s, x_{m}(s, z, \eta)\right) d s, \\
& \Lambda_{m}(z, \eta):=\int_{a}^{b}\left[g\left(s, x_{m}(s, z, \eta)\right)+h\left(s, f\left(s, x_{m}(s, z, \eta)\right)\right)\right] d s-d .
\end{aligned}
$$

Note that, unlike (4.7) and (4.8), equations (5.1) and (5.2) can be constructed explicitly.

In view of Theorem 1, it is natural to expect that, under suitable conditions, systems (4.7), (4.8) and (5.1), (5.2) are close enough to one another for $m$ sufficiently large.

Lemma 1. Assume that the conditions of Theorem 1 are satisfied and, moreover, $g \in \operatorname{Lip}_{K_{g}}(D)$ and $h \in \operatorname{Lip}_{K_{h}}(\tilde{D})$, where

$$
\tilde{D}:=\{f(t, y): t \in[a, b], y \in D\},
$$

with some non-negative square matrices $K_{g}$ and $K_{h}$ of dimension $n$. Then the exact and approximatathe determining functions defined by (4.4), (4.9) and (5.3), (5.2) satisfy the following estimates for any $(z, \eta) \in D_{a} \times D_{b}$ and $m \geq 1$ :

$$
\begin{aligned}
& \left|\Delta(z, \eta)-\Delta_{m}(z, \eta)\right| \leq \frac{10(b-a)^{2}}{27} K Q^{m}\left(\mathbb{1}_{n}-Q\right)^{-1} \delta_{[a, b], D}(f) \\
& \left|\Lambda(z, \eta)-\Lambda_{m}(z, \eta)\right| \leq \frac{10(b-a)^{2}}{27}\left(K_{g}+K_{h} K\right) Q^{m}\left(\mathbb{1}_{n}-Q\right)^{-1} \delta_{[a, b], D}(f),
\end{aligned}
$$

where the matrix $Q$ and the vector $\delta_{[a, b], D}(f)$ are given in (3.4) and (2.4) respectively.

Proof. Let us fix an arbitrary $(z, \eta) \in D_{a} \times D_{b}$. Using the Lipschitz condition (2.3), estimate (4.5) and the equality

$$
\int_{a}^{b} \alpha_{1}(t, a, b) d t=\frac{(b-a)^{2}}{3}
$$


where $\alpha_{1}(\cdot, a, b)$ is given in (4.6), we have

$$
\begin{aligned}
\left|\Delta(z, \eta)-\Delta_{m}(z, \eta)\right| & =\left|\int_{a}^{b} f\left(s, x_{m}(s, z, \eta)\right) d s-\int_{a}^{b} f\left(s, x_{\infty}(s, z, \eta)\right) d s\right| \\
& \leq K \int_{a}^{b} \frac{10}{9} \alpha_{1}(s, a, b) Q^{m}\left(\mathbb{1}_{n}-Q\right)^{-1} \delta_{[a, b], D}(f) d s \\
& =\frac{10(b-a)^{2}}{27} K Q^{m}\left(\mathbb{1}_{n}-Q\right)^{-1} \delta_{[a, b], D}(f),
\end{aligned}
$$

which proves the inequality in (5.6).

From (4.8) and (5.2) using the Lipschitz condition for $g$ and the relations (4.5), (5.8) we obtain

$$
\begin{aligned}
\left|\Lambda(z, \eta)-\Lambda_{m}(z, \eta)\right| & =\mid \int_{a}^{b}\left[g\left(s, x_{\infty}(s, z, \eta)\right)+h\left(s, f\left(s, x_{\infty}(s, z, \eta)\right)\right)\right. \\
& \left.-g\left(s, x_{m}(s, z, \eta)\right)-h\left(s, f\left(s, x_{m}(s, z, \eta)\right)\right)\right] d s \mid \\
\leq & K_{g} \int_{a}^{b}\left|x_{\infty}(s, z, \eta)-x_{m}(s, z, \eta)\right| d s \\
& +K_{h} K \int_{a}^{b}\left|x_{\infty}(s, z, \eta)-x_{m}(s, z, \eta)\right| d s \\
\leq & \left(K_{g}+K_{h} K\right) \int_{a}^{b} \frac{10}{9} \alpha_{1}(s, a, b) Q^{m}\left(\mathbb{1}_{n}-Q\right)^{-1} \delta_{[a, b], D}(f) d s \\
\leq & \frac{10(b-a)^{2}}{27}\left(K_{g}+K_{h} K\right) Q^{m}\left(\mathbb{1}_{n}-Q\right)^{-1} \delta_{[a, b], D}(f),
\end{aligned}
$$

that is, estimate (5.7) also holds.

Based on the exact an approximate determining systems (4.7), , (4.8) and (5.1), (5.2), let us fix an $m$ and introduce the mappings $H: D_{a} \times D_{b} \rightarrow \mathbb{R}^{2 n}$ and $H_{m}: D_{a} \times D_{b} \rightarrow \mathbb{R}^{2 n}$ by setting

$$
H(z, \eta):=\left(\begin{array}{c}
\eta-z-\int_{a}^{b} f\left(s, x_{\infty}(s, z, \eta)\right) d s \\
\int_{a}^{b}\left[g\left(s, x_{\infty}(s, z, \eta)\right)+h\left(s, f\left(s, x_{\infty}(s, z, \eta)\right)\right)\right] d s-d
\end{array}\right)
$$

and

$$
H_{m}(z, \eta):=\left(\begin{array}{c}
\eta-z-\int_{a}^{b} f\left(s, x_{m}(s, z, \eta)\right) d s \\
\int_{a}^{b}\left[g\left(s, x_{m}(s, z, \eta)\right)+h\left(s, f\left(s, x_{m}(s, z, \eta)\right)\right)\right] d s-d
\end{array}\right)
$$


for any $(z, \eta) \in D_{a} \times D_{b}$. Recall that $x_{\infty}\left(\cdot, z^{*}, \eta^{*}\right)$ is the limit function of the recurrence sequence (4.1).

We see from Theorem 2 that the critical points of the vector field $H$ determine solutions of the integral boundary value problem (3.1), (3.2). The next statement establishes a similar result based upon properties of the vector field $H_{m}$ explicity known from (5.10).

Theorem 4. Assume that the conditions of Lemma 1 hold. Let, moreover, one can specify an $m \geq 1$ and a set $\Omega \subset \mathbb{R}^{2 n}$ of form

$$
\Omega:=D_{1} \times D_{2}
$$

where $D_{1} \subset D_{a}, D_{2} \subset D_{b}$ are certain bounded open sets such that the mapping $H_{m}$ satisfies the relation

$$
\left|H_{m}\right| \triangleright_{\partial \Omega} \frac{10(b-a)^{2}}{27}\left(\begin{array}{c}
K Q^{m}\left(\mathbb{1}_{n}-Q\right)^{-1} \delta_{[a, b], D}(f) \\
\left(K_{g}+K_{h} K\right) Q^{m}\left(\mathbb{1}_{n}-Q\right)^{-1} \delta_{[a, b], D}(f)
\end{array}\right)
$$

on $\partial \Omega$. If, in addition,

$$
\operatorname{deg}\left(H_{m}, \Omega, 0\right) \neq 0,
$$

then there exists a pair $\left(z^{*}, \eta^{*}\right)$ from $D_{1} \times D_{2}$ for which the function

$$
x^{*}(\cdot):=x_{\infty}\left(\cdot, z^{*}, \eta^{*}\right)
$$

is a solution of the integral boundary value problem (3.1), (3.2).

In (5.12), $\partial \Omega$ is the boundary of $\Omega$ and the binary relation $\triangleright_{\partial \Omega}$ is defined [2] as follows: functions $u=\left(u_{i}\right)_{i=1}^{2 n}: \mathbb{R}^{2 n} \rightarrow \mathbb{R}^{2 n}$ and $v=\left(v_{i}\right)_{i=1}^{2 n}: \mathbb{R}^{2 n} \rightarrow \mathbb{R}^{2 n}$ are said to satisfy the relation $u \triangleright_{\partial \Omega} v$ if and only if there exists a function $k: \partial \Omega \rightarrow$ $\{1,2, \ldots, 2 n\}$ such that $u_{k(z)}(z)>v_{k(z)}(z)$ at every point $z \in \partial \Omega$.

Remark 3. The degree in (5.13) is the Brouwer degree because all the vectors fields are finite-dimensional. Likewise, all the terms in the right-hand side of (5.12) are computed explicitly (e.g., by using computer algebra systems).

Proof of Theorem 4. We shall use Lemma 1 stated above. By analogy to [2, 4, 8], we shall prove that the vector fields $H$ and $H_{m}$, given by (5.9) and (5.10) are homotopic. For this purpose, we consider the linear deformation determined by the family of mappings

$$
\Psi_{\vartheta}(z, \eta)=H_{m}(z, \eta)+\vartheta\left[H(z, \eta)-H_{m}(z, \eta)\right], \quad(z, \eta) \in \partial \Omega,
$$

for $\vartheta \in[0,1]$. Obviously, $\Psi_{\vartheta}$ is a continuous mapping on $\partial \Omega$ for every $\vartheta \in[0,1]$ and, moreover,

$$
\Psi_{0}=H_{m}, \quad \Psi_{1}=H
$$

on $\partial \Omega$. 
For arbitrary $\vartheta \in[0,1]$ and $(z, \eta) \in \partial \Omega$, we have

$$
\begin{aligned}
\left|\Psi_{\vartheta}(z, \eta)\right| & =\left|H_{m}(z, \eta)+\vartheta\left[H(z, \eta)-H_{m}(z, \eta)\right]\right| \\
& \geq \mid H_{m}\left(z, \eta|-| H(z, \eta)-H_{m}(z, \eta) \mid .\right.
\end{aligned}
$$

On the other hand, by estimates (5.6) and (5.7) of Lemma 1, we have the componentwise inequalities

$$
\left.\mid H(z, \eta)-H_{m}(z, \eta)\right) \mid \leq \frac{10(b-a)^{2}}{27}\left(\begin{array}{c}
K Q^{m}\left(\mathbb{1}_{n}-Q\right)^{-1} \delta_{[a, b], D}(f), \\
\left(K_{g}+K_{h} K\right) Q^{m}\left(\mathbb{1}_{n}-Q\right)^{-1} \delta_{[a, b], D}(f)
\end{array}\right) .
$$

Therefore, it follows from (5.12) and (5.16) that

$$
\left|\Psi_{\vartheta}\right| \triangleright_{\partial \Omega} 0
$$

for any $\vartheta \in[0,1]$. Here, 0 stands for the zero column vector of dimension $2 n$. Relation (5.17) implies that $\Psi_{\vartheta}$ dos not vanish on $\partial \Omega$ for any $\theta$, i. e., deformation (5.15) is non-degenerate. Thus, $H$ is homotopic to $H_{m}$ and, using assumption (5.13) and the property of invariance of the Brouwer degree under homotopy, we conclude that

$$
\operatorname{deg}(H, \Omega, 0)=\operatorname{deg}\left(H_{m}, \Omega, 0\right) \neq 0 .
$$

The classical topological result (see, e.g., [1, Theorem A2.4]) then guarantees the existence of a pair $\left(z^{*}, \eta^{*}\right) \in \Omega$ satisfying the equation $H\left(z^{*}, \eta^{*}\right)=0$. Therefore, the pair $\left(z^{*}, \eta^{*}\right)$ satisfies the system of determining equations (4.7), (4.8).

Applying now Theorem 2, we find that the function (5.14) is an absolutely continuous solution of integral boundary value problem (3.1), (3.2).

Remark 4. Theorem 1 does not guarantee the convergence of sequence (4.1) without assumption (3.5). In the case where condition (3.5) for the matrix $Q$ given in (3.4) does not hold, the limitation can be overcome by using a suitable parametrization and applying the interval halving technique introduced in $[5,8]$ for periodic boundary value problems.

Using this approach, the smallness condition (3.5) can be weakened to the inequality

$$
r\left(\frac{3(b-a)}{20} K\right)<1
$$

\section{SCHEME OF ANALYSIS OF THE PROBLEM}

on Theorem 4 consists in carrying out the following:

1. Choose the vector $\rho$ and compute the vector $\delta_{[a, b], D}(f)$ according to (2.4).

2. Check the fulfilment of inequality (3.3), construct the function $x_{m}(\cdot, z, \eta)$ analytically (e.g., by using computer algebra systems) for a certain fixed value $m=m_{0}$, keeping $z$ and $\eta$ as parameters. 
3. Select a suitable set $\Omega$ and verify conditions (5.12), (5.13) for $m=m_{0}$.

To verify condition (5.12) of Theorem 4 one has to use the reccurence formula (4.1) to compute the functions $x_{m}(\cdot, z, \eta)$ analytically, depending on the parameters $z$ and $\eta$, and, at every point $(z, \eta)$ of $\partial \Omega$, verify whether at least one component of the $2 n$-dimensional vector $\left|H_{m}(z, \eta)\right|$ is strictly greater than the corresponding component of the vector at the right-hand side of (5.12).

4. Verify the validity of (5.13).

This is rather difficult problem in general. However, there are sufficient conditions applicable in a number of important cases. In the smooth case, it follows directly from the definition of the topological degree [1, Definition A2.1] that if the Jacobian matrix of the function $H_{m}$ in (5.10) is non-singular at its isolated zero $\left(\bar{z}_{m}, \bar{\eta}_{m}\right)$, i. e.,

$$
\operatorname{det} \frac{\partial}{\partial(z, \eta)} H_{m}\left(\bar{z}_{m}, \bar{\eta}_{m}\right) \neq 0
$$

then inequality (5.13) holds. In (6.1), the symbol $\partial / \partial(z, \eta)$ means the derivative with respect to the vector of variables $\left(z_{1}, \ldots, z_{n}, \eta_{1}, \ldots, \eta_{n}\right)$.

In particular, when $H_{m}$ is odd mapping, i.e.

$$
H_{m}(-z,-\eta)=-H_{m}(z, \eta)
$$

for all $(z, \eta) \in \Omega$, then, according to Borsuk theorem (see, e. g., [1, Theorem A2.12]), its Brouwer degree is an odd number and, therefore, is different from zero.

Theorem 1 can be complemented by the following natural observation. Let $(\hat{z}, \hat{\eta}) \in$ $D_{a} \times D_{b}$ be a root of the approximate determining system (5.1), (5.2) for a certain $m$. The observation in Section 5 suggests to consider $\hat{z}$ and $\hat{\eta}$ as approximations of the values of a solution of problem (3.1), (3.2), respectively, at $a$ and $b$. Furthermore, the function

$$
\tilde{x}(t):=x_{m}(t, \hat{z}, \hat{\eta}), \quad t \in[a, b],
$$

defined according to (4.1) can be regarded as the $m$ th approximation to a solution of the integral boundary value problem (3.1), (3.2). The latter is justified by the estimate

$$
\left.\mid x_{\infty}(t, \hat{z}, \hat{\eta})-x_{m}(t, \hat{z}, \hat{\eta})\right) \mid \leq \frac{10}{9} \alpha_{1}(t, a, b) Q^{m}\left(\mathbb{1}_{n}-Q\right)^{-1} \delta_{[a, b], D}(f)
$$

implied directly by inequality (4.5) of Theorem 1 for any $t \in[a, b]$ and $m \geq 0$. Recall that, in (6.3), $Q$ and $\delta_{[a, b], D}(f)$ are given by (3.4) and (2.4) respectively. 


\section{AN EXAMPLE With TWO-SOLUTIONS}

Let us apply the numerical-analytic approach desribed above to the system of differential equations

$$
\left\{\begin{array}{l}
x_{1}^{\prime}(t)=x_{2}^{2}(t)-\frac{t}{5} x_{1}(t)+\frac{t^{3}}{100}-\frac{t^{2}}{25} \\
x_{2}^{\prime}(t)=\frac{t^{2}}{10} x_{2}(t)+\frac{t}{8} x_{1}(t)-\frac{21}{800} t^{3}+\frac{1}{16} t+\frac{1}{5}, \quad t \in\left[0, \frac{1}{2}\right],
\end{array}\right.
$$

considered under the integral boundary conditions

$$
\left\{\begin{aligned}
\int_{0}^{\frac{1}{2}} s x_{1}(s) x_{2}(s) d s & =-\frac{197}{48000} \\
\int_{0}^{\frac{1}{2}} s^{2} x_{2}^{2}(s) d s & =\frac{1}{4000} .
\end{aligned}\right.
$$

This boundary value problem was considered in [9]. Clearly, (7.1) is a particular case of (3.1), (3.2) with $a:=0, b:=1 / 2$,

$$
\begin{aligned}
& f\left(t, x_{1}, x_{2}\right):=\left(\begin{array}{c}
x_{2}^{2}-\frac{t}{5} x_{1}+\frac{t^{3}}{100}-\frac{t^{2}}{25} \\
\frac{t^{2}}{10} x_{2}+\frac{t}{8}\left(x_{1}+\frac{1}{2}\right)-\frac{21}{800} t^{3}+\frac{1}{5}
\end{array}\right), \\
& g\left(t, x_{1}, x_{2}\right):=\left(\begin{array}{c}
t x_{1} x_{2} \\
t^{2} x_{2}^{2}
\end{array}\right), h:=0, \text { and } d:=\left(\begin{array}{c}
-197 / 48000 \\
1 / 4000
\end{array}\right) .
\end{aligned}
$$

Following to (3.6), (3.7), introduce the parameters $z=\operatorname{col}\left(z_{1}, z_{2}\right)$ and $\eta=\operatorname{col}\left(\eta_{1}, \eta_{2}\right)$. Let us consider the following choice of the convex subsets $D_{a}$ and $D_{b}$, where one looks the values $x(a)$ and $x(b)$ :

$$
D_{a}=D_{b}=\left\{\left(x_{1}, x_{2}\right):-0.55 \leq x_{1} \leq 0.45,-0.2 \leq x_{2} \leq 0.15\right\} .
$$

In this case, according to (2.1), we have

$$
D_{a, b}=D_{a}=D_{b} \text {. }
$$

For $\rho$ involved in (2.2) and (3.3), we choose the value

$$
\rho:=\operatorname{col}(0.2,0.2) \text {. }
$$

Then, in view of (7.4)-(7.6), the set (2.2) takes the form

$$
D=\left\{\left(x_{1}, x_{2}\right):-0.75 \leq x_{1} \leq 0.65,-0.4 \leq x_{2} \leq 0.35\right\}
$$

A direct computation shows that the Lipschitz condition (2.3) for $f$ given by (7.3) on $D$ of form (7.7) holds with

$$
K=\left(\begin{array}{ll}
1 / 10 & 9 / 10 \\
1 / 16 & 1 / 40
\end{array}\right)
$$


and, therefore, by (3.4), $Q=\frac{3}{20}\left(\begin{array}{cc}1 / 10 & 9 / 10 \\ 1 / 16 & 1 / 40\end{array}\right)$ and $r(Q)=0.045<1$. Furthermore, in view of (7.7) and (2.4),

$$
\delta_{[a, b], D}(f)=\frac{1}{2}\left(\max _{(t, x) \in\left[0, \frac{1}{2}\right] \times D} f(t, x)-\min _{(t, x) \in\left[0, \frac{1}{2}\right] \times D} f(t, x)\right)=\left(\begin{array}{c}
0.15 \\
0.053125
\end{array}\right)
$$

and, by (7.6),

$$
\frac{b-a}{2} \delta_{[a, b], D}(f)=\left(\begin{array}{c}
0.0375 \\
0.01328125
\end{array}\right) \leq \rho .
$$

Note that $f$ is continuous.

We thus see that all the conditions of Theorem 1 are fulfilled, and the sequence of functions (4.1) for this example is convergent.

Using (4.1) and applying Maple 13 at the first iteration $(m=1)$ we get [9]

$$
\begin{aligned}
& x_{11}(t, z, \eta)= z_{1}+\frac{t^{4}}{400}+\frac{1}{3}\left(\left(-2 z_{2}+2 \eta_{2}\right)^{2}+\frac{2}{5}\left(z_{1}-\eta_{1}\right)-\frac{1}{25}\right) t^{3} \\
&+\frac{1}{2}\left(2 z_{2}\left(-2 z_{2}+2 \eta_{2}\right)-\frac{1}{5} z_{1}\right) t^{2}+z_{2}^{2} t-2 t\left(-\frac{29}{19200}\right. \\
&+\frac{1}{24}\left(-2 z_{2}+2 \eta_{2}\right)^{2}-\frac{1}{120} z_{1}-\frac{1}{60} \eta_{1} \\
&\left.+\frac{1}{4} z_{2}\left(-2 z_{2}+2 \eta_{2}\right)+\frac{1}{2} z_{2}^{2}\right)+2 t\left(\eta_{1}-z_{1}\right)
\end{aligned}
$$

and

$$
\begin{aligned}
x_{12}(t, z, \eta)=z_{2}+\frac{1}{5} t & +\frac{1}{4}\left(\frac{1}{5}\left(\eta_{2}-z_{2}\right)-\frac{21}{800}\right) t^{4} \\
+\frac{1}{3}\left(\frac{1}{4}\left(\eta_{1}-z_{1}\right)+\frac{1}{10} z_{2}\right) t^{3}+\frac{1}{16}\left(z_{1}+\frac{1}{2}\right) t^{2} & -2 t\left(\frac{5499}{51200}+\frac{1}{960} z_{2}+\frac{1}{320} \eta_{2}\right. \\
& \left.+\frac{1}{192} z_{1}+\frac{1}{96} \eta_{1}\right)+2 t\left(\eta_{2}-z_{2}\right),
\end{aligned}
$$

where we use the notation $x_{m i}=\operatorname{col}\left(x_{m, 1}, x_{m, 2}\right)$ for any $m$. Using (7.8) and (7.9) to form the approximate determining system (5.1), (5.2) for $m=1$ and solving the latter numerically, we find its roots $z_{1}=\operatorname{col}\left(z_{11}, z_{12}\right)$ and $\eta_{1}=\operatorname{col}\left(\eta_{11}, \eta_{12}\right)$ :

$$
\begin{aligned}
& z_{11} \approx-0.5000145056, \quad z_{12} \approx 5.750026703 \cdot 10^{-7}, \\
& \eta_{11} \approx-0.4875143149, \quad \eta_{12} \approx 0.1000004007 .
\end{aligned}
$$


Substituting (7.10) into (7.8), we obtain the first and second components of the first approximation $\tilde{x}_{1}=\operatorname{col}\left(\tilde{x}_{11}, \tilde{x}_{12}\right)$ to a solution of the given integral boundary value problem (7.1), (7.2):

$$
\begin{aligned}
\tilde{x}_{11}(t)= & -0.5000145056+(1 / 400) t^{4}-0.001666738533 t^{3} \\
& +0.05000156555 t^{2}+0.00010378326 t, \\
\tilde{x}_{12}(t)= & 5.750026703 \cdot 10^{-7}+0.1999349926 t-0.001562508715 t^{4} \\
& +0.0010417017 t^{3}-9.066 \cdot 10^{-7} t^{2} .
\end{aligned}
$$

Let us now show that problem (7.1), (7.2) indeed has a solution in a neighbourhood of $\tilde{x}_{1}$. For this purpose, we analyse the corresponding properties of first approximation given in (7.8) and use Theorem 4.

We choose the domain $\Omega:=D_{1} \times D_{2}$ in (5.11) as a direct product of rectangles

$$
\begin{aligned}
& D_{1}:=\left\{\left(z_{1}, z_{2}\right):-0.55 \leq z_{1} \leq 0.1 ;-0.1 \leq z_{2} \leq 0.05\right\} \subset D_{a}, \\
& D_{2}:=\left\{\left(\eta_{1}, \eta_{2}\right):-0.55 \leq \eta_{1} \leq 0 ; 0 \leq \eta_{2} \leq 0.12\right\} \subset D_{b} .
\end{aligned}
$$

The the boundaries $\partial D_{1}$ and $\partial D_{2}$ thus have, respectively, the equations

$$
\begin{aligned}
& \left\{z_{1}=-0.55, \quad-0.1 \leq z_{2} \leq 0.05\right\}, \\
& \left\{z_{2}=0.05, \quad-0.55 \leq z_{1} \leq 0.1\right\} \\
& \left\{z_{1}=0.1, \quad-0.1 \leq z_{2} \leq 0.05\right\} \\
& \left\{z_{2}=-0.1, \quad-0.55 \leq z_{1} \leq 0.1\right\}
\end{aligned}
$$

and

$$
\begin{aligned}
& \left\{\eta_{1}=-0.55, \quad 0 \leq \eta_{2} \leq 0.12\right\}, \\
& \left\{\eta_{2}=0.12, \quad-0.55 \leq \eta_{1} \leq 0\right\}, \\
& \left\{\eta_{1}=0, \quad 0 \leq \eta_{2} \leq 0.12\right\}, \\
& \left\{\eta_{2}=0, \quad-0.55 \leq \eta_{1} \leq 0\right\} .
\end{aligned}
$$

Consequently, the fulfilment of the relation (5.12) for the first iteration $(m=1)$ should be checked for all values (7.14) and (7.15).

Direct computations for this example give that $g \in \operatorname{Lip}_{K_{g}}(D)$ with $D$ given by (7.7) and

$$
K_{g}=\left(\begin{array}{cc}
0.2 & 0.375 \\
0 & 0.2
\end{array}\right)
$$

Furthermore,

$$
\frac{10(b-a)^{2}}{27}\left(\begin{array}{c}
K Q\left(\mathbb{1}_{n}-Q\right)^{-1} \delta_{[a, b], D}(f) \\
K_{g} Q\left(\mathbb{1}_{n}-Q\right)^{-1} \delta_{[a, b], D}(f)
\end{array}\right)=\left(\begin{array}{c}
2.32707024 \cdot 10^{-4} \\
6.065016891 \cdot 10^{-4} \\
2.406173922 \cdot 10^{-4} \\
3.155040783 \cdot 10^{-5}
\end{array}\right) .
$$


Comparing the terms involved in (5.12) at the points determined by equations (7.14) and (7.15) and using (7.16), we find that relation (5.12) is satisfied in this case.

In order to verify condition (5.13), we use the differentiability of $f$. We form the Jacobi matrix

$$
\frac{\partial H_{1}}{\partial(z, \eta)}=\left(\begin{array}{llll}
\frac{\partial H_{11}}{\partial z_{1}} & \frac{\partial H_{11}}{\partial z_{2}} & \frac{\partial H_{11}}{\partial \eta_{1}} & \frac{\partial H_{11}}{\partial \eta_{2}} \\
\frac{\partial H_{12}}{\partial z_{1}} & \frac{\partial H_{12}}{\partial z_{2}} & \frac{\partial H_{12}}{\partial \eta_{1}} & \frac{\partial H_{12}}{\partial \eta_{2}} \\
\frac{\partial H_{13}}{\partial z_{1}} & \frac{\partial H_{13}}{\partial z_{2}} & \frac{\partial H_{13}}{\partial \eta_{1}} & \frac{\partial H_{13}}{\partial \eta_{2}} \\
\frac{\partial H_{14}}{\partial z_{1}} & \frac{\partial H_{14}}{\partial z_{2}} & \frac{\partial H_{14}}{\partial \eta_{1}} & \frac{\partial H_{14}}{\partial \eta_{2}}
\end{array}\right)
$$

where

$$
\begin{aligned}
& H_{11}(z, \eta)=\eta_{1}-z_{1}-\int_{a}^{b} f_{1}\left(t, x_{11}(t, z, \eta), x_{12}(t, z, \eta)\right) d t \\
& H_{12}(z, \eta)=\eta_{2}-z_{2}-\int_{a}^{b} f_{2}\left(t, x_{11}(t, z, \eta), x_{12}(t, z, \eta)\right) d t, \\
& H_{13}(z, \eta)=\int_{a}^{b} g_{1}\left(t, x_{11}(t, z, \eta), x_{12}(t, z, \eta)\right) d t+\frac{197}{48000}, \\
& H_{14}(z, \eta)=\int_{a}^{b} g_{2}\left(t, x_{11}(t, z, \eta), x_{12}(t, z, \eta)\right) d t-\frac{1}{4000}
\end{aligned}
$$

with $f$ and $g$ as above. At point (7.10), matrix (7.17) has the value

$$
\left(\begin{array}{cccc}
-0.991664946 & 0.0165680208 & 1.016639475 & 0.0334006242 \\
-0.005205078 & -1.001032832 & -0.0104752604 & 0.9967402959 \\
-0.00208135681 & -0.02066615277 & -0.00609620114 & -0.041047519 \\
-0.00000185526 & -0.00124495269 & 0.00001482908 & 0.0049824817
\end{array}\right) .
$$

The determinant of the above matrix is equal to $5.0813401 \cdot 10^{-5}$ and, in particular, is different from 0. Recalling Section 6, we conclude that (6.1) holds with $\bar{z}_{1}=$ $\operatorname{col}\left(z_{11}, z_{12}\right)$ and $\bar{\eta}_{1}=\operatorname{col}\left(\eta_{11}, \eta_{12}\right)$ given by (7.10), whence (5.13) follows. Thus, by virtue of Theorem 4 , there exist a pair $\left(z^{*}, \eta^{*}\right) \in D_{1} \times D_{2}$ such that function (5.14) is a solution of the integral boundary value problem (7.1), (7.2).

It is easy to verify that the pair of functions

$$
x_{1}^{*}(t)=\frac{t^{2}}{20}-\frac{1}{2}, \quad x_{2}^{*}(t)=\frac{t}{5}
$$

is a solution of the integral boundary value problem (7.1), (7.2) and its values at 0 and $1 / 2$,

$$
\begin{gathered}
x_{1}^{*}(0)=-0.5, \quad x_{2}^{*}(0)=0, \\
x_{1}^{*}\left(\frac{1}{2}\right)=-\frac{39}{80}, \quad x_{2}^{*}\left(\frac{1}{2}\right)=\frac{1}{10},
\end{gathered}
$$

belong to the respective sets $D_{1}$ and $D_{2}$ of form (7.12) and (7.13). 
Comparing (7.11) with (7.18), we find that the error of the first approximation is estimated as

$$
\max _{t \in\left[0, \frac{1}{2}\right]}\left|x_{1}^{*}(t)-x_{11}(t)\right| \leq 2 \cdot 10^{-5}, \quad \max _{t \in\left[0, \frac{1}{2}\right]}\left|x_{2}^{*}(t)-x_{12}(t)\right|=6 \cdot 10^{-6} .
$$

The graphs of the first approximation and the exact solution of the given boundary value problem are shown on Figure 1.
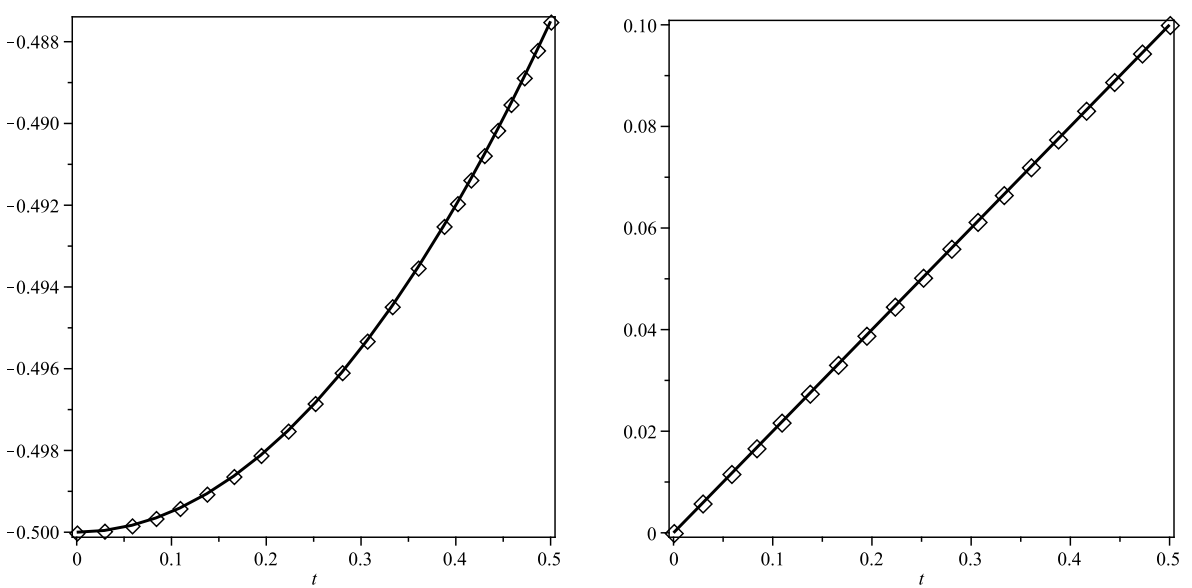

FIGURE 1. The components of the exact solution (solid line) and its first approximation (drawn with dots)

According to Theorems 2 and 3, the number of solutions of the algebraic detemining system (4.7), (4.8) coincides with the number of solutions of the given integral boundary value problem in the domain under consideration. Computations show that, along with solution (7.10), the approximate determining system of algebraic equations (5.1), (5.2) for $m=1$ has another solution

$$
\begin{aligned}
& \hat{z}_{11} \approx 0.3923536713, \quad \hat{z}_{12} \approx-0.1570525052, \\
& \hat{\eta}_{11} \approx 0.3868493960, \quad \hat{\eta}_{12} \approx-0.04383992217 .
\end{aligned}
$$

Instead of $\Omega:=D_{1} \times D_{2}$ defined by (7.12), (7.13), we now choose $\tilde{\Omega}=D_{3} \times D_{4}$ with

$$
\begin{aligned}
& D_{3}=\left\{\left(z_{1}, z_{2}\right): 0 \leq z_{1} \leq 0.42,-0.2 \leq z_{2} \leq-0.12\right\}, \\
& D_{4}=\left\{\left(\eta_{1}, \eta_{2}\right): 0 \leq \eta_{1} \leq 0.41,-0.1 \leq \eta_{2} \leq 0.05\right\} .
\end{aligned}
$$


The equations of $\partial D_{3}$ and $\partial D_{4}$ then have the form

$$
\begin{aligned}
& \left\{z_{1}=0,\right. & -0.2 & \left.\leq z_{2} \leq-0.12\right\}, \\
& \left\{z_{2}=-0.12,\right. & 0 & \left.\leq z_{1} \leq 0.42\right\}, \\
& \left\{z_{1}=0.42,\right. & -0.2 & \left.\leq z_{2} \leq-0.12\right\}, \\
& \left\{z_{2}=-0.2,\right. & 0 & \left.\leq z_{1} \leq 0.42\right\}
\end{aligned}
$$

and

$$
\begin{aligned}
\left\{\eta_{1}=0,\right. & -0.1 & \left.\leq \eta_{2} \leq 0.05\right\}, \\
\left\{\eta_{2}=0.05,\right. & 0 & \left.\leq \eta_{1} \leq 0.41\right\}, \\
\left\{\eta_{1}=0.41,\right. & -0.1 & \left.\leq \eta_{2} \leq 0.05\right\}, \\
\left\{\eta_{2}=-0.1,\right. & 0 & \left.\leq \eta_{1} \leq 0.41\right\} .
\end{aligned}
$$

Checking (5.12) for the first iteration of form (7.8) at the boundary points (7.22) and (7.23), we find that (5.12) is satisfied. A direct computation shows that (5.13) also holds. Consequently, by Theorem 4 , there exist a pair $\left(\hat{z}^{*}, \hat{\eta}^{*}\right) \in D_{3} \times D_{4}$ such that the function

$$
\hat{x}^{*}(t)=x_{\infty}\left(t, \hat{z}^{*}, \hat{\eta}^{*}\right), \quad t \in[0,1 / 2],
$$

is a solution of the integral boundary value problem (7.1), (7.2) with $\left(\hat{x}_{1}^{*}(0), \hat{x}_{2}^{*}(0)\right) \in$ $D_{3},\left(\hat{x}_{1}^{*}(1 / 2), \hat{x}_{2}^{*}(1 / 2) \in D_{4}\right.$. By substituting (7.19) into the first iteration (7.8), we obtain the following first approximation to solution (7.24) of (7.1), (7.2):

$$
\begin{aligned}
\hat{x}_{11}(t)= & 0.3923536713+(1 / 400) t^{4}+0.004490021967 t^{3} \\
& -0.07479600670 t^{2}+0.02495444725 t, \\
\hat{x}_{12}(t)= & -0.1570525052+0.2000752910 t-0.0009018708475 t^{4} \\
& -0.005693773113 t^{3}+0.05577210445 t^{2} .
\end{aligned}
$$

We see that the values of (7.24) at 0 and $1 / 2$,

$$
\begin{aligned}
\hat{x}_{11}(0) & =0.39235367135, & \hat{x}_{12}(0) & =-0.1570525052, \\
\hat{x}_{11}\left(\frac{1}{2}\right) & =0.3868493959, & \hat{x}_{12}\left(\frac{1}{2}\right) & =-0.04383992216
\end{aligned}
$$

belong to $D_{3}$ and $D_{4}$ of form (7.20) and (7.21) respectively. By analogy, we can obtain the second and third approximations $(m=2,3)$ to solution (7.24).

The residual obtained as a result of substitution of the third approximation to solution (7.24) into the given differential system (7.1) is estimated as follows:

$$
\begin{array}{r}
\max _{t \in\left[0, \frac{1}{2}\right]}\left|x_{31}^{\prime}(t)-x_{32}^{2}(t)+\frac{t}{5} x_{31}(t)-\frac{t^{3}}{100}+\frac{t^{2}}{25}\right|=3.230806 \cdot 10^{-8}, \\
\max _{t \in\left[0, \frac{1}{2}\right]}\left|x_{32}^{\prime}(t)-\frac{t^{2}}{10} x_{32}(t)-\frac{t}{8} x_{31}(t)+\frac{21}{800} t^{3}-\frac{1}{16} t-\frac{1}{5}\right|
\end{array}
$$


The graphs of first and third approximations to the second solution of the given boundary value problem are shown on Figure 2.
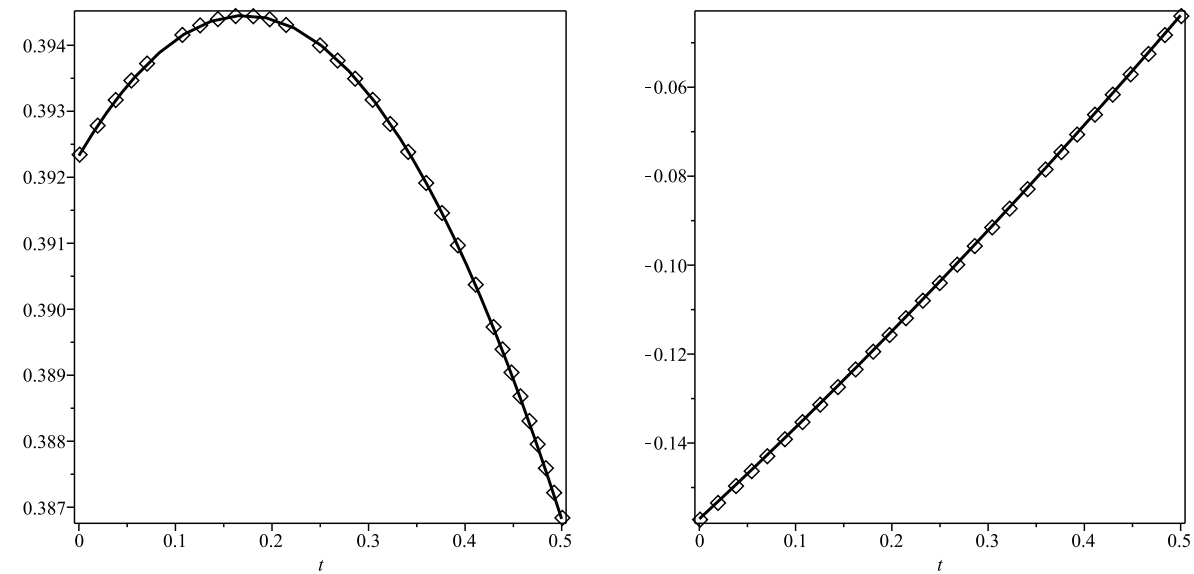

FIGURE 2. The components of the first (drawn with dots) and third (solid line) approximations to the second solution

\section{REFERENCES}

[1] M. Farkas, Periodic motions, ser. Applied Mathematical Sciences. New York: Springer-Verlag, 1994, vol. 104

[2] A. Rontó and M. Rontó, "Successive approximation techniques in non-linear boundary value problems for ordinary differential equations," in Handbook of differential equations: ordinary differential equations. Vol. IV, ser. Handb. Differ. Equ. Elsevier/North-Holland, Amsterdam, 2008, pp. 441-592.

[3] A. Rontó and M. Rontó, "On nonseparated three-point boundary value problems for linear functional differential equations," Abstr. Appl. Anal., pp. Art. ID 326052, 22, 2011. [Online]. Available: http://dx.doi.org/10.1155/2011/326052

[4] A. Rontó and M. Rontó, "Existence results for three-point boundary value problems for systems of linear functional differential equations," Carpathian J. Math., vol. 28, no. 1, pp. 163-182, 2012.

[5] A. Rontó and M. Rontó, "Periodic successive approximations and interval halving," Miskolc Math. Notes, vol. 13, no. 2, pp. 459-482, 2012.

[6] A. Rontó and M. Rontó, "On constructive investigation of a class of non-linear boundary value problems for functional differential equations," Carpathian J. Math., vol. 29, no. 1, pp. 91-108, 2013.

[7] A. Rontó, M. Rontó, G. Holubová, and P. Nečesal, "Numerical-analytic technique for investigation of solutions of some nonlinear equations with Dirichlet conditions," Bound. Value Probl., pp. 1-20, 58, 2011. [Online]. Available: http://dx.doi.org/10.1186/1687-2770-2011-58

[8] A. Rontó, M. Rontó, and N. Shchobak, "Constructive analysis of periodic solutions with interval halving," Bound. Value Probl., pp. 2013:57, 34, 2013. [Online]. Available: http://dx.doi.org/10.1186/1687-2770-2013-57 
[9] A. Rontó, M. Rontó, and Y. Varha, "A new approach to non-local boundary value problems for ordinary differential systems," Applied Mathematics and Computation, vol. 250, pp. 689-700, 2015.

[10] M. Rontó and K. Marinets, "On the parametrization of boundary value problems with two-point nonlinear boundary conditions," Nelīnünn̄ Koliv., vol. 14, no. 3, pp. 359-391, 2011.

[11] M. Rontó and K. Marynets, "On the parametrization of boundary-value problems with three-point non-linear restrictions," Miskolc Math. Notes, vol. 13, no. 1, pp. 91-106, 2012.

[12] M. Rontó and A. M. Samoilenko, Numerical-analytic methods in the theory of boundary-value problems. River Edge, NJ: World Scientific Publishing Co. Inc., 2000, with a preface by Yu. A. Mitropolsky and an appendix by the authors and S. I. Trofimchuk. [Online]. Available: http://dx.doi.org/10.1142/9789812813602

[13] M. Rontó, Y. Varha, and K. Marynets, "Further results on the investigation of solutions of integral boundary value problems," Tatra Mt. Math. Publ., submitted.

[14] M. Rontó and K. Marynets, "Parametrization for non-linear problems with integral boundary conditions," Electron. J. Qual. Theory Differ. Equ., no. 99, pp. 1-23, 2012.

[15] M. Rontó and N. Shchobak, "On parametrization for a non-linear boundary value problem with separated conditions,” Electron. J. Qual. Theory Differ. Equ., vol. 8, no. 18, pp. 1-16, 2007.

Authors' addresses

Miklós Rontó

Department of Analysis, University of Miskolc, 3515 Miskolc-Egyetemváros

E-mail address: matronto@uni-miskolc.hu

\section{Yana Varha}

Uzhgorod National University, 14 Universitetska St., 88000 Uzhgorod, Ukraine

E-mail address: jana.varha@mail.ru 